\title{
The new bone formation and microstructure assessed by combination of confocal laser scanning microscopy and differential interference contrast microscopy
}

\author{
Xiaohong Yang ${ }^{1,2^{*}}$ Ling Qin ${ }^{2,3}$ Weiguo Liang ${ }^{1}$ Wen Wang ${ }^{1}$ Jianrong Tan $^{1}$ \\ Peihong Liang ${ }^{1}$ Jiake $\mathrm{Xu}^{4^{*}}$ Siming $\mathrm{Li}^{1}$ Shuliang Cui ${ }^{1,5^{*}}$
}

1 Guangzhou Institute of Traumatic Surgery, The Fourth Affiliated Hospital, Jinan University School of Medicine, Guangzhou 510220, China

2 Musculoskeletal Research Laboratory, Department of Orthopaedics and Traumatology, Chinese University of Hong Kong, Shatin, NT, Hong Kong SAR, China

3 Translational Medicine Research \& Development Center, Institute of Biomedical and Health Engineering, Shenzhen Institutes of Advanced Technology, Chinese Academy of Sciences, Shenzhen 518055, China

4 School of Pathology and Laboratory Medicine, University of Western Australia, Crawley Western Australia 6009, Australia

5 Department of Zoology, University of Melbourne, Parkville Victoria 3010, Australia

Receive correspondence :

Xiaohong Yang

Guangzhou Institute of Traumatic Surgery

The Fourth Affiliated Hospital

Jinan University School of Medicine

396 Tong Fu Zhonglu Road

Hai Zhu District, Guangzhou, Guangdong Province

P. R. China, 510220

Phone Number: 862034403731

Fax Number: 862034403824

Email: dryang1192@tom.com

\footnotetext{
* Corresponding to: Xiaohong Yang, Guangzhou Institute of Traumatic Surgery, The Fourth Affiliated Hospital, Jinan University School of Medicine, Guangdong Province 510220, P. R. China, dryang1192@tom.com; Jiake Xu, School of Pathology and Laboratory Medicine, University of Western Australia, WA 6009, Australia. jiake.xu@uwa.edu.au; Shuliang Cui, Department of Zoology, University of Melbourne, Victoria 3010, Australia. s.cui2008@gmail.com
} 


\begin{abstract}
Bone is a mineralized connective tissue that is continuously and micro-structurally remodelled. Altered bone formation and microstructure arise in pathological bone diseases such as osteoporosis, osteonecrosis, fracture repair and Paget's disease of bone. A proper and objective assessment of bone formation and microstructure will provide insights into the understanding of bone pathogenesis and remodeling. Here, the new bone formation ex vitro and its microstructure was evaluated in in vivo multiple sequential polychrome-labelled samples using confocal laser scanning microscopy (CLSM), which generated clearer and more reliable images of thick bone sections than conventional fluorescence microscopy (CFM). Intriguingly, fine details of the bone microstructural features, including the mineralization fronts, quiescent versus active osteons, and Volkmann's channel, were elucidated using CLSM, which defines relationship between morphological changes and function, when combined with differential interference contrast (DIC) microscopy. Furthermore, CLSM provided objective evaluations of bone formation, such as the ratio of labelled areas of new bone formation in a rabbit model when compared with CFM. Altogether, new bone formation and its microstructure can be evaluated more adequately using combination of the CLSM and DIC microscopies.
\end{abstract}

Keywords Confocal laser scanning microscopy; Fluorescence labeling; Differential interference contrast microscope; New bone formation; Microstructure.

Running tittle: Dynamic imaging of new bone formation by CLSM and CFM

\title{
Introduction
}

Bone is a mineralized connective tissue that contains cortical and trabecular bone and is continuously and microstructurally remodelled under physiological conditions. Abnormal bone formation and microstructure arise in pathological bone diseases. A more precise progressive evaluation of bone formation and microstructure will facilitate the diagnosis and treatment of bone diseases.

The mineralization front of bony ossification surfaces in new bone formation are commonly labelled using tetracycline and other fluorescent agents. The bone apposition rate in new bone formation can be 
calculated when polychrome sequential labeling is employed by in vivo administration of fluorescent agents, either orally or intravenously [1-4]. Relatively large histological thick sections of $100-300 \mu \mathrm{m}$ are prepared from non-decalcified bone embedded in methymethacrylate or/and series of thin sections (8$20 \mu \mathrm{m})$, which require a special heavy-duty microtome for preparation in conjunction with the process of evaluation of the specimens, is time consuming [5-7], are technically inevitably needed for achieving quality images to visualize the fluorescent labeling under the conventional florescent microscope (CFM) for analysis without partial volume effect (PVE), i.e. images are hampered by the loss or underestimation of apparent activity in small objects/regions from the finite spatial resolution of imaging systems where detected photons originating at locations within the tissue are blurred into surrounding locations in close proximity during data acquisition $[8,9]$.

Confocal laser scanning microscopy (CLSM) is a technique for obtaining high-resolution optical images through process of controlled optical sectioning with highly limited depth of focus by selection, only one depth level at a time and, assisted with a computer, point-by-point to reconstruct 3D images of topologically complex objects with non-superimposed interior images at multiple depths over conventional microscopy, which visualizes through as the light penetrates [10]. CLSM enables the 3D assessment of the cell-matrix information layer-by-layer in a non-invasive manner for thick tissue samples without mechanical sectioning $[11,12]$. Sequential images were obtained from rat samples of transforming growth factor (TGF- $\beta$ ) implant for promoting new bone formation in the cranium and iliac bone and used to calculate the rate of bone formation [12]; Comparisons of sections of unstained fresh samples and Hematoxylin \& Eosin (H\&E) stained sections were made between CFM and CLSM [13].

Study using CLSM on the mineralization of articular cartilage in race horses simultaneously detected two important parameters with high repetitiveness; including the linear growth rate and fluorescence density [14]. Bisphosphonates and related drugs, such as risedronate, and its lower-affinity analogues deoxyrisedronate and 3-(3-Pyridyl)-2-hydroxy-2-phosphonopropanoic acid (3-PEHPC), bind to calcium in different affinities for bone mineral at resorbing surfaces and forming surfaces, which differs from that observed at quiescent surfaces. The distribution of fluorescent labelled calcium-binders of various affinities were detected, revealed the effects of their bone mineral affinity within the 
skeleton [15]. Moreover, pulsed with calcein (CAL), alizarin complexone (AL), and tetracycline (TC), bone formation around hydroxyapatite (HA) implants in normal and with diabetes mellitus (DM) animals at various times after implantation was scanned with CLSM and showed different bone formation patterns in normal group from DM group [16]. Using dual staining of fluorescent calcein and alizarin red at different times, temporal patterns of bone formation were accurately assessed by CLSM in undecalcified thick sections with titanium implants left in situ and profiled the bone first formed as thin processes towards and across the implant surface followed by further development with a space in the bone-implant interface [17]. Overlapped with high-resolution imaging of surfaces with compositional information acquired by Back-scattered Electron Detector (BSE) combined with Scanning Electron Microscopy (SEM), CLSM scanning filled the gap of cells and osteoid left by BSE to rebuild cellular localization in bone samples embedded in polymethylmethacrylate [18].

CLSM was used quantitatively in single-molecule imaging based on fluorescence intensity distribution for statistically analysis of molecular occurrence in different forms, including DNA and proteins in solution [19]. CLSM was also used for subcellular analysis in fungi [20] for host-fungus interactions and spatial patterns of colonization, and for obtaining sharp images of fungi within a host or colonizing highly contoured or rugged surfaces [21]. Fluorescence of a visible wavelength Ca2+-sensitive fluorophore in isolated cardiac myocytes was visualized using CLSM as a measurement of intracellular calcium changes occurring in spontaneously active cardiac cells [22].

3D structure imaging and quantitative characterization in thick tissue based on CSLM and derivative techniques were used in quantitative imaging of biomolecular localization and trafficking in live cells [23]. Fluorescence signals in cartilage of cryostat sections of mouse articular cartilage were quantified by localizing and quantifying insulin-like growth factor-1 (IGF-1) receptor expression in situ using CLSM to analyse its distribution patterns in chondrocytes [24], which was further applied in quantitative analysis of in situ localization patterns of IGF-1 receptor in murine normal or arthritic intact patellae model [25]. These results showed the powerful capability of CLSM in elucidating cellular 
structure and function of bone tissues. When CLSM is linked to DIC, it is technically possible to obtain clear fluorescence-labeled images with the assistance of transmitted light for an accurate localization of the fluorescence-labeled dye on the bone surface.

In this study, the new bone formation and bone microstructure, including the mineralization fronts, quiescent versus active osteons, and Volkmann's channel, in thick bone samples were assayed using DIC combined with the CLSM to generate fluorescent images of high resolution that could be used in quantitative assessing the progressive mineral deposition of bone growth.

\section{Materials and methods}

\section{Polychrome sequential labelling of bone in vivo}

The fluorescent agents calcein green (excitation $488 \mathrm{~nm}$ ) and xylenol orange (excitation 453nm) (Sigma) were prepared in phosphate buffer $(\mathrm{pH}$ 7.2), sterilized and filtered for in vivo injection at a dose of 10 $\mathrm{mg} / \mathrm{kg}$ and $90 \mathrm{mg} / \mathrm{kg}$, respectively.

Four adult female Chinese goats, $26 \pm 3.2 \mathrm{~kg}$, were injected with fluorescent dyes intravenously once every two weeks for four times prior to sample collection, with an injection sequence of calcein green $\rightarrow$ xylenol orange $\rightarrow$ calcein green $\rightarrow$ xylenol orange [26]. Longitudinal sections of tibia were prepared to observe the effects of multiple fluorescent labeling in images acquired by CLSM. Transected samples were also prepared to investigate the relation between bone surface structure and bone mineral deposition labeled with fluorescence using DIC.

A partial patellectomy model in rabbits was employed as previously described [27] to assess the bone formation using CFM and CLSM. Four 18-week-old adult female New Zealand rabbits, $3.5 \pm 0.5 \mathrm{~kg}$ of body weight, were anaesthetized using $10 \%$ ketamine $(0.5 \mathrm{ml} / \mathrm{kg})$ i.m. and $2.5 \mathrm{ml} 2.5 \%$ phenobarbitone sodium i.v. then the 1/3 distal end of woven bone was transversely sectioned and removed, two $0.8 \mathrm{~mm}$ pores were perforated vertically to the proximal end of residual woven bone. The patellar ligament was 
directly stitched onto the proximal woven bone with non-absorbable suture, which was protected with " 8 " steel thread, and fixed in position using gypsum for 6 weeks with the position of the knee joint at 90 degree flexion. The operation sites were given low-intensity pulsed ultrasound (LIPUS) treatment at day 3 after the operation using the physical parameters as detailed previously [27-29], to enhance the bone healing up to the $12^{\text {th }}$ week after operation. Following the surgery, the animals received calcein green and xylenol orange, s.c., once every 2 weeks and euthanized at 3 days after the last injection, woven bones were longitudinally sectioned for evaluation of the optical density of mineralized areas and quantitation of new bone formation rate. Animal ethics approval was obtained from The Fourth Affiliated Hospital, Jinan University School of Medicine prior to the experiments.

\section{Sample preparations}

All samples were fixed in 10\% neutral formalin solution, dehydrated in a graded series of ethanol and embedded in methyl methacrylate. Bone specimens were sectioned using a saw microtome (Leica SP1600, Germany) at a thickness of $400 \mu \mathrm{m}$ for goat samples and at a thickness of $300 \mu \mathrm{m}$ for rabbit samples according to our established protocol [30].

\section{Conventional fluorescent microscopy (CFM)}

Fluorescent images were obtained using a fluorescent microscope (Zeiss Axiovert 200M, Germany) equipped with a high voltage mercury lamp, a green broadband filter disk (\#9, Zeiss) and a red broadband filter disk (\#15, Zeiss). The overall histological structure of bone tissues was viewed under low magnification $(1.25 \times)$ and the fluorescence labelled line of calcification fronts was observed under high magnification (40×) using the Digital Color CCD (Charge Coupled Device) and Digital Video (Camera AxioCam MRc Rev. 2, 1388×1040, Zeiss). The tissue structure was displayed and evaluated using the Microscopic Image Analysis System (AxioVision Rel. 4.5 Software, Zeiss).

Confocal laser scanning microscopy system (CLSM) 
Images were acquired using the Multi-Tracking Scan (MTS) mode and MTS with transmitted light mode (Zeiss LSM 510 META system, Germany) with excitation wave lengths of $488 \mathrm{~nm}$ and $453 \mathrm{~nm}$ were selected for calcein green and xylenol orange, respectively; and the emission wave length of 520nm and $615 \mathrm{~nm}$ for calcein green and xylenol orange respectively as previously described [5];

In MTS mode, single layer images were acquired using dual fluorescent tracks of green for calcein green and red for xylenol orange, and analysed using the conventional histomorphometric method of bone remodelling or apposition rate, which measures the distance between two fluorescent labels generated by timed injection and divided by the number of days between injections to give the rise of an average growth rate, i.e. distance $(\mu \mathrm{m}) /$ days $[1,2,31]$, and the estimation of new bone formation, in which region of interest (ROI) was selected using the image analysis software for quantifying two different fluorescent dyes by calculating mean fluorescence-labeled areas for calcein green and xylenol orange as reported index of new bone formation rate [27]. The estimation of new bone formation in repairs was carried out by comparisons of the areas $(\mu \mathrm{m} \times \mu \mathrm{m})$ of red and green fluorescence labels in the ROI obtained using the image analysis software (AIM, Carl Zeiss, LSM 510 Release Version 4.0 SP2), in which the fluorescent dyes were quantified in pixels as mean intensity with a standard derivation and converted into areas, to those in the control group so that the background noises is eliminated. The ratio of the mean fluorescence labeled area by calcein green over the mean area labeled by xylenol orange was used as bone formation rates in NB region of patellectomy repairing in this study.

Thick samples were optically sectioned in ROI to obtain 131 continuous images of $225 \mu \mathrm{m}(\mathrm{X}) \times 225 \mu \mathrm{m}$ (Y) $\times 0.51 \mu \mathrm{m}(\mathrm{Z})$, and those images were sequentially overlapped along the Z-axis with a suggested optimal interval of 0.5 to form a stack of $225 \mu \mathrm{m}(\mathrm{X}) \times 225 \mu \mathrm{m}(\mathrm{Y}) \times 65.99 \mu \mathrm{m}(\mathrm{Z})$ in CLSM using a laser pointing beam with no damages to the block, which allowed the real three-dimensional reconstruction (as detailed in Zeiss Operating Manual, Carl Zeiss MicroImaging GmbH, Göttingen, Germany). The images 
were manipulated and viewed using the CLSM image analysis software Physiology/Timeseries for Release 4.0.

\section{Statistical analysis}

One-way ANOVA analysis in the SPSS13.0 software package was adopted to perform the statistical analyses of the digitized quantitative data.

\section{Results}

\section{Comparative imaging analysis of bone formation by CFM and CLSM}

Comparison of fluorescent dye signals in images of thick sections of goat tibial bone that received 4 sequential injections of calcein green $\rightarrow$ xylenol orange $\rightarrow$ calcein green $\rightarrow$ xylenol orange, showed clear differences between CFM and CLSM. In the image acquired by CFM with >100 X magnification, indistinct connection of fluorescent labels accompanied by intercrossing and interfering signals was observed above and below the focal plane of the labeled bands in a typical section (Fig. 1a), which gave blurry labeling lines of the calcification front. In the same ROI, sharp labeling lines from the four distinct dye bands with no interfering in the image acquired by CLSM (Fig. 1b), indicating that the imaging quality of fluorescent-labeled thick bone sections generated by CLSM was much higher than those by CFM.

The CLSM optical scanning with the thickness of $0.51 \mu \mathrm{m}$ was adjusted along the longitudinal section of goat tibial bone in depth of $65.99 \mu \mathrm{m}$ to obtain images with required signal brightness of fluorescent labels [32]. The bone tissue block was scanned by CLSM in a ROI of $225 \mu \mathrm{m}(\mathrm{X}) \times 225 \mu \mathrm{m}(\mathrm{Y})$ from the surface layer to the deep layer to generate sequential single optical microscopic sections (Fig. 1b). The sectioning was moved longitudinally ( $\mathrm{Z}$ axis) across a whole osteon with an optimal interval of 0.5 to obtain optical section images for $3 \mathrm{D}$ reconstruction showing the spatial structure of the bone formed (Fig. 1c). The measurement of thick bone sections represented new bone formed within the ROI of whole samples with 
no limitation just to the surface layer. By dragging a cross mark at the center of the figure to any position on the image and the depth of calcification labels in the sample block was showed by inserted thumbnails on top and side figures (Fig. 1d), which illustrated the distribution of deposited calcium in the process of bone formation in 3D that cannot be done by CFM.

Improved assessment of bone formation by CFM and CLSM using a partial patellectomy model in rabbits

Patella is a sesamoid bone embedded within a ligament in the knee joint and maintains in a quiescent state for bone mineral apposition in adult rabbits normally, with only both ends attached to the tendon displaying fluorescent labels of the mineral deposition as observed by CFM under low magnification (Fig. 2a). Twelve weeks after the partial patellectomy in the LIPUS treatment, the patella was in an exuberant bone metabolic process, especially in new bone forming area where the fluorescent labeled mineral became more obvious, indicative of increased bone remodeling activity as visualized by CFM (Fig. 2b). Moreover, the newly formed bone was labeled in stronger green than overlapped red labels (Fig. 2f-h) while the fluorescent labeling in the old bone area displayed a regular pattern of fewer-overlapped green and red (Fig. 2i-k). A substantially overlapped dual-colored labeling bands were seen by CLSM scanning in the new bone formation (Fig. 2h), but few locations were visible across the region with the widest point of $20.85 \mu \mathrm{m}$ (Fig. 2c, e). The new bone growth rate according to the traditional measurement of distance was calculated as $20.85 \mu \mathrm{m} \div 14 \mathrm{~d}=1.49 \mu \mathrm{m} / \mathrm{d}$. Multiple locations of dual-colored mineral labels with the widest point of $34.31 \mu \mathrm{m}$ (Fig. 2d, e) were recorded in the old bone region and its growth rate was calculated as $34.31 \mu \mathrm{m} \div 14 \mathrm{~d}=2.45 \mu \mathrm{m} / \mathrm{d}$, which is higher than that of the new bone formation. It is noted that there seem to be irregular patterns of fluorescent labeling in new bone tissues. The new bone growth rate was obtained by comparison of the calcein green and xylenol orange labeled areas in images acquired by CLSM with coordinately adjustment of operating parameters, such as the laser power of transmission, pinhole diameter, thickness of optical sectioning and detector gain, for conformational evaluation of the new bone growth rate (Fig. 21). The requited new bone growth rate agreed with the intensity of fluorescent labels observed by microscopy. 


\section{Improved visualization of bone microstructure by CLSM in the combination of differential interference contrast (DIC) microscopy}

The MTS images of fluorescence labeling signals were obtained by CLSM (Fig. 3a, b), and coalesced by DIC transmitted light images to accurately localize the microstructure of the labeled bone matrix for fluorescence measurement. The mineralization fronts of osteons were localized in bone samples and their relationship with bony structures around the fluorescence labels were clearly shown with static and active osteons visible simultaneously within ROI (Fig. 3c). Quiescent osteons were observed with no fluorescent signals while active osteons were labeled with dual fluorescence. The images were merged to show their activeness of mineral calcification timely and, notably, Volkmann's channels were also visualized in compact bone, and run perpendicularly to the Haversian canals (Fig. 3d).

\section{Discussion}

CLSM is an advanced imaging technology developed in the 1980s, and has gradually become popular in all biological and medical research fields. CLSM uses a laser as a light source with penetration ability based on optical confocal technology combined with a laser scanning device attached to the fluorescence microscope to obtain images that are manipulated by a computer to produce higher quality images than the CFM. The application of laser, confocal imaging and pinhole prevented from interferes of diffracted light and scattered light, their MTS function ensures enough fluorescent signals, especially weak fluorescent light signals, and eliminated cross talks between samples so that MTS scan samples with multiple labels at the same time through multiple channels, the fluorescent signals are further processed using specific software to filter out the effects of "cross talks". When the optical "sections" of nondecalcified thick bone with sequential polychromes were observed using the CLSM, the laser scanning microscope generated sharp images, which were able to solve the problem of PVEs encountered when using CFM (Fig. 1). The images by MTS were verified by the META, which is a special mode in Zeiss LSM 510 recognizing signals by specific pattern of individual wave length for acquiring pixels, to eliminate "cross talks" between fluorescent signals. Although section thickness less than $10 \mu \mathrm{m}$ was previously reported for sharp images by CFM [33], sectioning of bone tissues are difficult without 
decalcification. Bone quantification studies by CFM requires sections of $\geq 10 \mu \mathrm{m}$ using a specific heavyduty microtome for clear images and the thin sections are easy broken, and it is practically difficult to prepare enough sequential sections at this thickness range for non-decalcified bone tissue sections.

The CLSM overcomes those difficulties by using thick sections. For bone samples with polychrome sequential labeling, the section thickness scanned by CLSM can be readily adjusted and is more advantageous than CFM, including, as it does, a wider selection of wave-length ranges, high sensitivity, high discriminability, strong contrast and sharp imaging. Upper and lateral specific ROI were observed simultaneously and the depth of the labeled mineralization in a sample layer were shown (Fig. 1d, e). These 3D structures were not readily visualized using CFM. Collectively, CLSM displays superior imaging quality of fluorescent-labeled bone sections, and provides an option of 3D imaging reconstruction. To date, CLSM obtains fluorescence labeled images with a range of wave-lengths simultaneously at high sensitivity to distinguish labels with narrow wave length differences $[34,35]$.

Normal osteoblasts secrete osteoid that will be mineralized layer by layer with collagen fiber forming pore-like structure aligning parallel to the mineral lamella of $7-10 \mu \mathrm{m}$ for calcium hydroxyapatite precipitation and crystallization in about 10 days under physiological conditions [11]. Due to the mineralization lag phenomenon, there is a band of osteoid that is formed between the mineralized bone surface and osteoblasts. In vivo, tetracycline and other florescent dyes will be deposited in the mineralization front of the ossification surface, the new bone formation being then labeled in the experimental animals treated orally and intravenously with fluorescent labeling agents several times [11]. Calcein green and xylenol orange can specifically bind to calcium, deposited on mineralization front and show bright green and red fluorescent colors under a fluorescence microscope respectively.

The fluorescence labelled area is related to the amount of combined calcium. Measurement of morphologic components includes directly measured static variables and kinetic variables usually generated by fluorescent labels applied at timed intervals and integrated into the forming bones. The distance between different labels reflects the rate of bone formation, and those in vivo traces generated by bone remodeling permit dynamically detection and estimation of rates of remodeling activity and active 
osteons [1]. Mineralization fronts within lamellae of the active osteons in cortical bone were visualized in thin sections by CFM, and their apposition rate presented as the distance between the fluorescent bands over the number of days after in vivo injection of polychrome fluorescence dyes, and was further used for the estimation of new bone formation rate in intact bones [1, 31]. CLSM observations (Fig. 2 c-e) in this study suggest that the conventional bone mineral apposition rate calculated by measuring the distance between two parallel labeling bands can be more precisely defined without PVE when compared with CFM in intact bone. However, the bone mineral apposition rate by distance measurement in healing bones was even slower than that for intact or old bone, suggesting a limitation in its application in studying healing bone mineral apposition. This finding was also consistent with other observations by CFM where the new bone formation rate was found to be far higher than that of the remodeling shown in old bones in our previous study [27]. There were no significant differences in bone apposition rate were detected during the bone repairing of sesamoid boneas compared to normal bone formation by the CFM-based measurement (Fig. 2e). In fact, the new bone formed around the residual proximal patella after partial patellectomy showed no regular bone plate structure and typical mineralization fronts by CLSM (Fig. f-h). The new bone growth after surgeon and fracture repair is rapid in overlapped reticular formats, showing blurred images under CFM (not effect of signal cross talks), which is different from the normal bone development and cannot form clearly separated decalcification bands. The conventional distance measurement approach is not applicable for evaluation of new bone formation and its remodeling in healing bones, e.g. in fracture repair where it undergoes rapid osteogenesis or new bone formation with irregular mineral deposition and integration of disorganized collagen fibers as shown in the partial patellectomy rabbit model of this study.

Images of fluorescent labels in the field of newly formed bone were acquired by CFM individually using filters in thin sections. The fluorescent signals were quantified and interpreted as ratio of labeled areas with xylenol orange and calcein green to study the dynamics of osteogenesis and its remodeling [6]. The quantification and calculation of labeled areas in images obtained using CLSM easily were more accurate on an optical section of thick tissues. Eventually, significant differences in labelled areas of calcein green and xylenol orange were detected (Fig. 21) between the new bone and the old bone. Altogether, ratio of labelled areas calculated by CLSM recorded differential outcomes on progressive new bone formation 
during repair of sesamoid boneas compared to normal bone formation, which is not readily appreciated by CFM-based technique. The CLSM allows to define the fluorescence labels in the lamellar diffusion type and linear marginal during new bone formation. The results of this work suggest the fluorescence intensity measurements as mean areas using CLSM is a better option to conventional distance measurement and the area ratio calculation for analyses of new bone formation in repairing.

DIC technology was originally used to observe the structure of histological sections without staining by the supplementation of a pair of polar elements and a Wollaston glass prism in the phase-contrast microscope to increase the contrast of interface characteristics of specimens $[11,36]$. The related data of the fluorescence image and the DIC image can be obtained in the same procedure, and the overlapped images are accurately distinguished by their repeatable confocal measurements [37]. Densities of bone samples can be precisely detected using X-ray and CT scanning, but those methods are not suitable for demonstrating the activeness of bone cells at a given developing stage. The investigations on thick nondecalcified bone samples are rarely reported previously. The calcification in vivo is a dynamic process, the number and density of labeled bands represent the levels of calcification at a particular time, which makes labels and density different even in the same sample with no labels for quiescent osteons at a particular developmental stage (Fig.3). This study used CLSM technique combined with DIC to localize the mineralized bone matrix labeled with multiple fluorescent dyes. In particular, the introduction of DIC technique to the study of the dynamics of osteons enables the precise localization of the mineralized front with polychrome sequential labeling in thick non-decalcified bone sections, not only localized the calcified labels but also revealed the relations between morphology and function according to the activeness of osteons. The beginning and the stages of new bone formation can be determined by color integrations. The application of fluorescent labeling technique combined with DIC in this report ascertains a combined microscopic interpretation for researches of bone development, which is instructively significant for thick bone samples compared to samples of conventional cell cultures. Further development of CLSM and the DIC technique might provide a new imaging processing protocol for the study of bone mineral metabolism and healing in calcified tissue research. 
In summary, the new bone formation and bone microstructure, including the mineralization fronts were investigated by CLSM, which advantaged in adjustments of operating parameters, thickness of optical sections and automatic image acquisition and analysis. Polychrome sequential fluorescence quantification is more accurate with higher repetitiveness than CFM and areas ratio of sequential fluorescent labels in new bone formation by CLSM in healing bone was calculated as new bone growth rate, a better estimation of bone growth in repairing. CLSM combined with DIC technique further generated sequentially fluorescent labeling images showing mineral apposition with details of microstructure, including osteons and Volkmann's channel. Presence or absence of fluorescent labels in osteons reflect their activeness during the bone formation and remodeling, which renders a new imaging process for the study of bone mineral metabolism and fracture healing.

\section{Acknowledgment}

This study was made possible by the financial support from Hong Kong RGC Earmarked Research Grant (CUHK: 4155/02M), Guangdong Natural Science Foundation Grant (S2012010010616), Key Project of Guangzhou Medical Health Science and Technology (20121A021009) and Science and Technology Project funded by the Guangzhou Municipal Government (2012J4100027). We are grateful to Dr. Tam KF for kindly making the bone samples available, Dr. Chen JW for advice on hard tissue sample preparation, both from the Department of Orthopaedics and Traumatology of the Chinese University of Hong Kong.

\section{References}

1. Eriksen EF, Axelrod DW, Melsen F (1994) Bone Histomorphometry. Raven Press, New York, USA

2. Mohsin S, O'Brien FJ, Lee TC (2006) Microcracks in compact bone: a three-dimensional view. Journal of anatomy 209:119-124

3. Lee TC, Arthur TL, Gibson LJ, Hayes WC (2000) Sequential labelling of microdamage in bone using chelating agents. Journal of orthopaedic research 18:322-325

4. Serra G, Morais LS, Elias CN, Meyers MA, Andrade L, Muller CA, Muller M (2010) Sequential bone healing of immediately loaded mini-implants: histomorphometric and fluorescence analysis. American journal of orthodontics and dentofacial orthopedics 137:80-90

5. Pautke C, Vogt S, Tischer T, Wexel G, Deppe H, Milz S, Schieker M, Kolk A (2005) Polychrome labeling of bone with seven different fluorochromes: enhancing fluorochrome discrimination by spectral image analysis. Bone 37:441-445

6. Lu H, Qin L, Fok P, Cheung W, Lee K, Guo X, Wong W, Leung K (2006) Low-intensity pulsed ultrasound accelerates bone-tendon junction healing: a partial patellectomy model in rabbits. American Journal of Sports Medicine 34:1287-1296 
7. Pautke C, Vogt S, Kreutzer K, Haczek C, Wexel G, Kolk A, Imhoff AB, Zitzelsberger H, Milz S, Tischer T (2010) Characterization of eight different tetracyclines: advances in fluorescence bone labeling. Journal of anatomy 217:76-82

8. Hoffman EJ, Huang SC, Phelps ME (1979) Quantitation in positron emission computed tomography: 1. Effect of object size. Journal of computer assisted tomography 3:299-308

9. Pretorius PH, King MA (2009) Diminishing the impact of the partial volume effect in cardiac SPECT perfusion imaging. Medical physics 36:105-115

10. Pawley JB (2006) Handbook of Biological Confocal Microscopy. Springer Science+Business Media, LLC, New York, USA

11. Li N, Yi L, Su Z (1997) Laser Scanning Confocal Microscopic Technology (in Chinese). People's Military Medical Press, Beijing, China

12. Okuda K, Nakajima Y, Irie K, Sugimoto M, Kabasawa Y, Yoshie H, Hara K, Ozawa H (1995) Transforming growth factor-beta 1 coated beta-tricalcium phosphate pellets stimulate healing of experimental bone defects of rat calvariae. Oral diseases 1:92-97

13. Grotz KA, Piepkorn B, Al-Nawas B, Duschner H, Bittinger F, Kann P, Beyer J, Wagner W (1999) Confocal laser scanning microscopy: A nondestructive subsurface histotomography of healthy human bone. Calcified tissue international 65:8-10

14. Doube M, Firth EC, Boyde A (2005) Registration of confocal scanning laser microscopy and quantitative backscattered electron images for the temporospatial quantification of mineralization density in 18-month old thoroughbred racehorse articular calcified cartilage. Scanning 27:219-226

15. Roelofs AJ, Stewart CA, Sun S, Blazewska KM, Kashemirov BA, McKenna CE, Russell RG, Rogers MJ, Lundy MW, Ebetino FH, Coxon FP (2012) Influence of bone affinity on the skeletal distribution of fluorescently labeled bisphosphonates in vivo. Journal of bone and mineral research 27:835-847

16. Iyama S, Takeshita F, Ayukawa Y, Kido MA, Suetsugu T, Tanaka T (1997) A study of the regional distribution of bone formed around hydroxyapatite implants in the tibiae of streptozotocin-induced diabetic rats using multiple fluorescent labeling and confocal laser scanning microscopy. Journal of periodontology 68:1169-1175

17. Nishikawa T, Masuno K, Mori M, Tajime Y, Kakudo K, Tanaka A (2006) Calcification at the interface between titanium implants and bone: observation with confocal laser scanning microscopy. The Journal of oral implantology 32:211-217

18. Boyde A, Lovicar L, Zamecnik J (2005) Combining confocal and BSE SEM imaging for bone block surfaces. European cells \& materials 9:33-38

19. Vukojević V, Heidkamp M, Ming Y, Johansson B, Terenius L, Rigler R (2008) Quantitative single-molecule imaging by confocal laser scanning microscopy. Proceedings of the National Academy of Sciences 105:18176-18181

20. Czymmek KJ, Whallon JH, Klomparens KL (1994) Confocal microscopy in mycological research. Experimental Mycology 18:275-293

21. Spear RN, Cullen D, Andrews JH (1999) Fluorescent labels, confocal microscopy, and quantitative image analysis in study of fungal biology. Methods in enzymology 307:607-623

22. Williams DA (1990) Quantitative intracellular calcium imaging with laser-scanning confocal microscopy. Cell Calcium 11:589-597

23. Raarup MK, Nyengaard JR (2011) Quantitative Confocal Laser Scanning Microscopy. Image Analysis and Stereology 25:111-120

24. Verschure PJ, van Marle J, Joosten LA, Van den Berg WB (1994) Localization and quantification of the insulin-like growth factor-1 receptor in mouse articular cartilage by confocal laser scanning microscopy. The journal of histochemistry and cytochemistry 42:765773

25. Verschure PJ, Van Marle J, Van Noorden CJ, Van den Berg WB (1997) The contribution of quantitative confocal laser scanning microscopy in cartilage research: chondrocyte insulin-like growth factor-1 receptors in health and pathology. Microscopy research and technique 37:285298

26. Tam KF, Cheung WH, Lee KM, Qin L, Leung KS (2009) Shockwave exerts osteogenic effect on osteoporotic bone in an ovariectomized goat model. Ultrasound in medicine \& biology 35:11091118 
27. Qin L, Fok P, Lu H, Shi S, Leng Y, Leung K (2006) Low intensity pulsed ultrasound increases the matrix hardness of the healing tissues at bone-tendon insertion-a partial patellectomy model in rabbits. Clinical biomechanics (Bristol, Avon) 21:387-394

28. Lui P, Zhang P, Chan K, Qin L (2010) Biology and augmentation of tendon-bone insertion repair. Journal of orthopaedic surgery and research 5:59

29. Lu H, Qin L, Lee K, Cheung W, Chan K, Leung K (2009) Identification of genes responsive to low-intensity pulsed ultrasound stimulations. Biochemical and biophysical research communications 378:569-573

30. Qin L, Mak AT, Cheng CW, Hung LK, Chan KM (1999) Histomorphological study on pattern of fluid movement in cortical bone in goats. The Anatomical record 255:380-387

31. Clark PA, Rodriguez A, Sumner DR, Hussain MA, Mao JJ (2005) Modulation of bone ingrowth of rabbit femur titanium implants by in vivo axial micromechanical loading. Journal of applied physiology 98:1922-1929

32. Zarrinkalam KH, Kuliwaba JS, Martin RB, Wallwork MA, Fazzalari NL (2005) New insights into the propagation of fatigue damage in cortical bone using confocal microscopy and chelating fluorochromes. European journal of morphology 42:81-90

33. Kazama JJ, Gejyo F, Ejiri S, Okada M, Ei I, Arakawa M, Ozawa H (1993) Application of confocal laser scanning microscopy to the observation of bone biopsy specimens. Bone 14:885889

34. Muller M (2006) Introduction to Confocal Fluorescence Microscopy Second Edition. SPIE The International Society for Potical Engineering, Washington, USA

35. NMatsumoto B (2002) Cell Biological Applications of Confocal Microscopy Second Edition. Academic Press, Elsevier Sciences, USA

36. Guo S, Luo X, Qiu G (2001) Base and Clin of Osteoporosis (in Chinese). Tianjin Science and Technology Press, Tianjin, China

37. Sahara M, Sata M, Matsuzaki Y, Tanaka K, Morita T, Hirata Y, Okano H, Nagai R (2005) Comparison of various bone marrow fractions in the ability to participate in vascular remodeling after mechanical injury. Stem cells (Dayton, Ohio) 23:874-878

Figure Legends

Figure 1. Comparative analysis of polychrome sequential labeled images by two different microscope systems. Longitudinal section of goat tibia with the thickness of $400 \mu \mathrm{m}$, scale $=50 \mu \mathrm{m}$. a: Conventional fluorescence microscope (CFM) image, with 4 broad and partially overlapped labelling lines that cannot be used for a precise quantification of bone apposition rate (measurement of the distance between lines). b: Confocal Laser Scanning Microscope (CLSM) image. Arrows 1-4 indicate labelling lines generated by four sequential intravenous fluorescent dye injections of calcein green $\rightarrow$ xylenol orange $\rightarrow$ calcein green $\rightarrow$ xylenol orange. c: A 3D image constructed by superimposing single layer optical sections acquired in $\mathbf{b}$. d: Orthogonal sectioning of $\mathbf{b}$, stacks in 3 cut views of xy, xz and yz. The $\mathrm{xz}$ image was upper flipped and the yz image was laterally flipped on the right of the xy image showing the mineralization detected in three dimensions. 
Figure 2. Analysis of bone tissue sections using CFM and CLSM. Rabbit patella after partial patellectomy was sagittally sectioned with the thickness of $300 \mu \mathrm{m}$. a, b: CFM image with low magnification of $12.5 \times$, and c-l: CLSM images. a: CFM image of a normal rabbit patella at a glance. b: CFM image of the patella 12 weeks after partial patellectomy (OB: old bone; NB: new bone, dash line shows original surgical cutting line of patellectomy, the region contains typical fluorescent labeling lines selected for calculating bone apposition rate is boxed in dash lines. c: Magnified NB box in b by CLSM showing the two clearly fluorescent labeled lines, the distance between the lines (white bar) was measured and used for calculation of bone apposition rate 12 weeks after the patellectomy. d: The magnified OB box in b by CLSM displaying the two fluorescent-labeled lines in old bone retained from the patellectomy, from which the distance (white bar) was measured for estimation of bone formation rate. e: Quantitative analysis of bone apposition rates for NB in $\mathbf{c}$ and $\mathrm{OB}$ in $\mathbf{d}$, calculated from the measured distances between labelled lines (white bars), which shows no significant difference statistically $(p>0.05)$. f-h: CLSM images of NB 12 weeks after patellectomy. i-k: CLSM images of OB at 12 weeks post patellectomy. $\mathbf{f}$ and $\mathbf{i}$ : CLSM images acquired using green fluorescence channel (calcein green). $\mathbf{g}$ and $\mathbf{j}$ : CLSM images acquired using red fluorescence channel (xylenol orange). $\mathbf{h}$ and $\mathbf{k}$ : Merged images. $\mathbf{l}$ : Differential analysis of the red/green ratio of labelled areas $\left(\mu \mathrm{m}^{2}\right)$ from control and patellectomy sample sections of 5 animals, demonstrating a highly significant difference between new bone and old bone $(* * p<0.01)$. Bar scale: $200 \mu \mathrm{m}$.

Figure 3. Fluorescent images acquired by CLSM plus DIC scanning. Crosscutting section of goat tibia with a thickness of $400 \mu \mathrm{m}$ in thick bone with a scale of $50 \mu \mathrm{m}$. a: Image acquired by green fluorescence channel. b: Image acquired by red fluorescence channel. c: Image acquired by DIC channel. d: Merged image of three-channel CLSM images (green and red fluorescence + DIC) of A, B and C. The merged image shows 2 calcified fluorescence labels from different times (green and red) plus DIC (grey). The weak red color from the second calcein labeling still exist and merged into first green labeling, combined into an yellowish color with various intensities depending upon the ratio of the two colors at developmental stages of those osteons. The arrow 1 indicates quiescent osteons in 
comparison to the fluorescently labeled active osteons; arrow 2 points out the Volkmann's channels. e: A diagrammatic illustration of the anatomical processing of bone specimens. f: Mean of total osteon numbers versus mean of the active osteon numbers showed on 10 images (views) from 5 animals at 20× magnification labeled with fluorescent dyes and acquired by CLSM combined with DIC. 

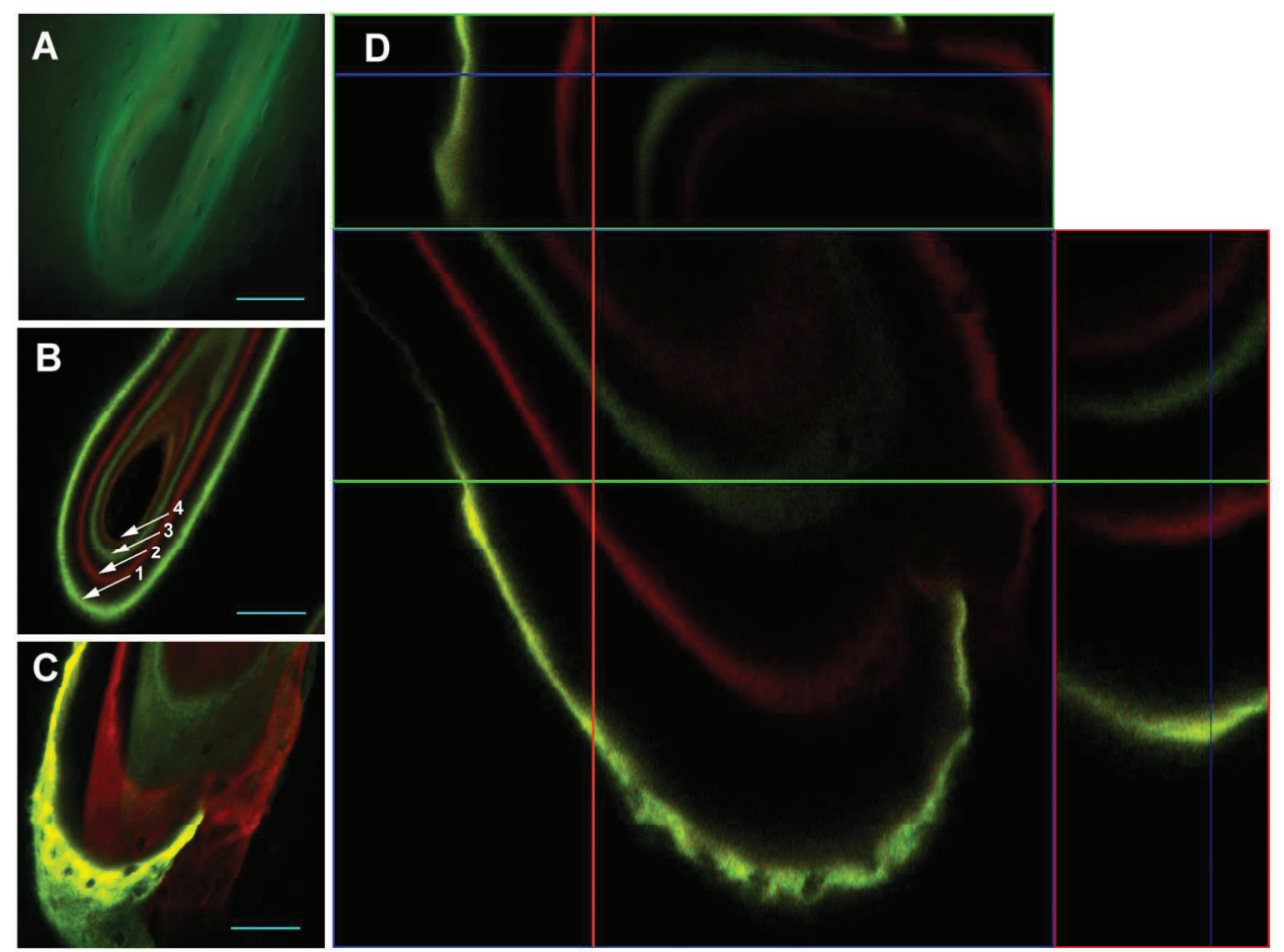

Figure 1.Comparative analysis of polychrome sequential labeledimages by two different microscope systems. Longitudinal section of goat tibia with the thickness of $400 \mu \mathrm{m}$, scale $=50 \mu \mathrm{m}$. a: Conventional fluorescence microscope (CFM) image, with 4 broad and partially overlapped labelling lines that cannot be used for a precise quantification of bone apposition rate (measurement of the distance between lines). b: Confocal Laser Scanning Microscope (CLSM) image. Arrows 1-4 indicate labelling lines generated by four sequential intravenous fluorescent dye injections of calcein green $\rightarrow$ xylenol orange $\rightarrow$ calcein green $\rightarrow$ xylenol orange. c: A 3D image constructed by superimposing single layer optical sections acquired in b. d: Orthogonal sectioning of $b$, stacks in 3 cut views of $x y, x z$ and $y z$. The $x z$ image was upper flipped and the $y z$ image was laterally flipped on the right of the $x y$ image showing the mineralization detected in three dimensions. $159 \times 120 \mathrm{~mm}(300 \times 300 \mathrm{DPI})$ 

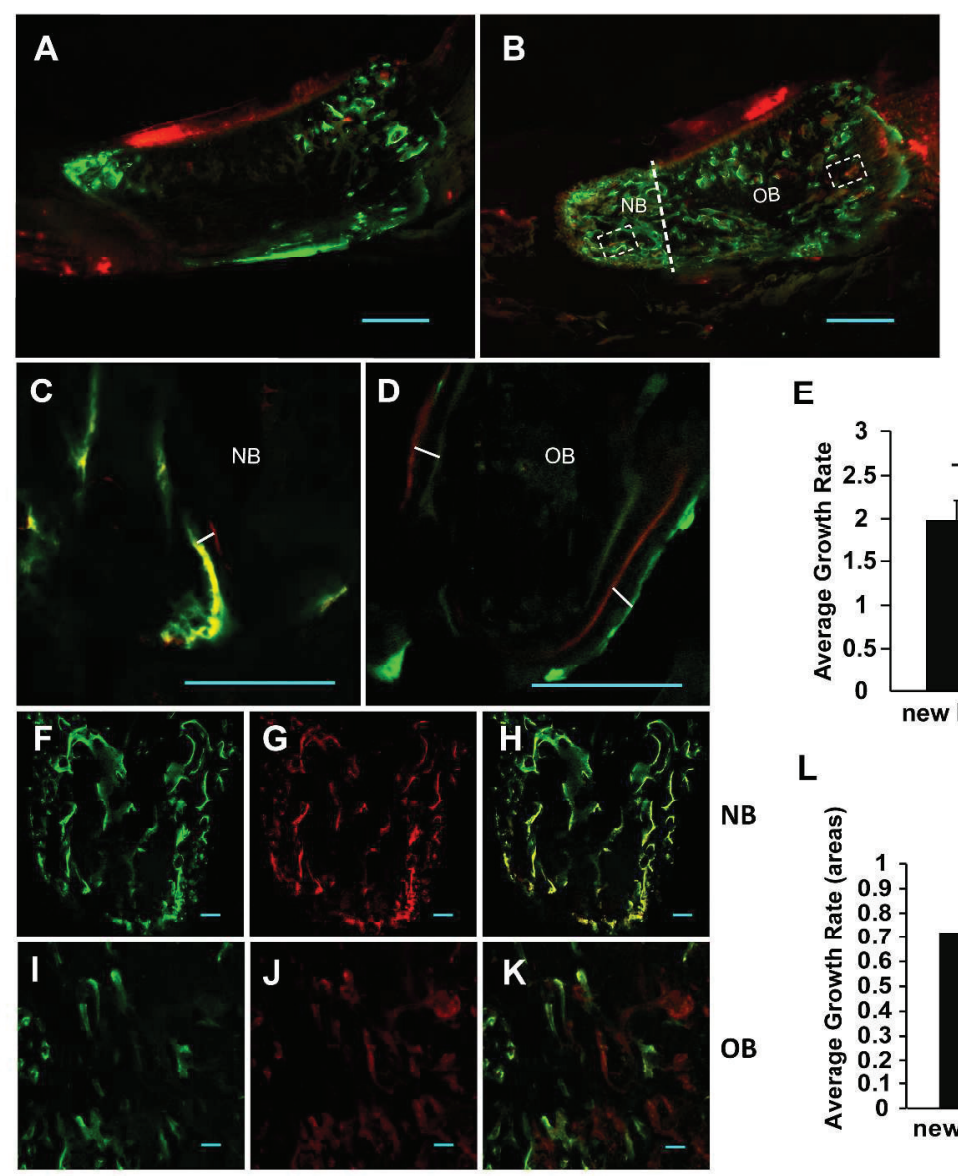

E
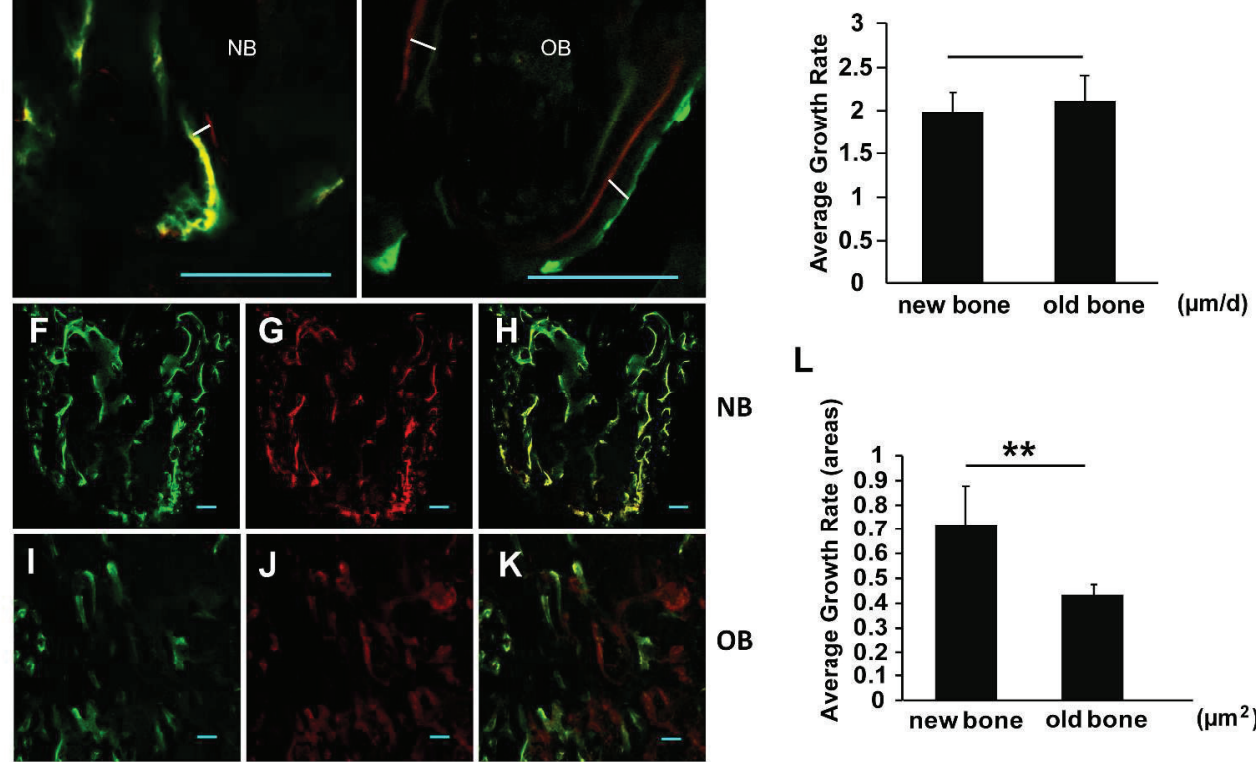

L

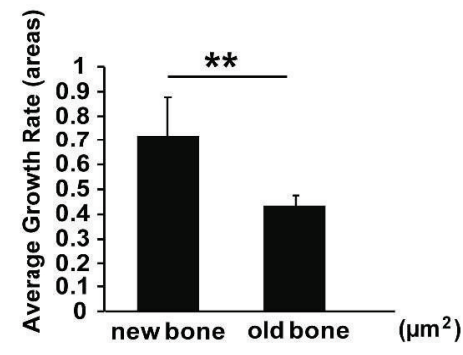

Figure 2. Analysis of bone tissue sections using CFM and CLSM. Rabbit patella after partial patellectomy was sagittally sectioned with the thickness of $300 \mu \mathrm{m}$. a, b: CFM image with low magnification of $12.5 \times$, and c-I: CLSM images. a: CFM image of a normal rabbit patellaat a glance. b: CFM image of the patella 12 weeks after partial patellectomy (OB: old bone; NB: new bone, dash line shows original surgical cutting line of patellectomy, the region contains typical fluorescent labeling lines selected for calculating bone apposition rate is boxed in dash lines. c: Magnified NB box in b by CLSM showing the two clearly fluorescent labeled lines, the distance between the lines (white bar) was measured and used for calculation of bone apposition rate 12 weeks after the patellectomy. d: The magnified OB box in b by CLSM displayingthe two fluorescentlabeled lines in old bone retained from the patellectomy, from which the distance (white bar) was measured for estimation of bone formation rate.e: Quantitative analysis of bone apposition rates for NB in $\mathrm{c}$ and OB ind, calculated from the measured distancesbetween labelled lines (white bars), which shows no significant difference statistically $(p>0.05)$. f-h: CLSM images of NB 12 weeks after patellectomy. i-k: CLSM images of OBat 12 weeks post patellectomy. $f$ and i: CLSM images acquired using green fluorescence channel (calcein green). $g$ and $\mathrm{j}$ : CLSM images acquired using red fluorescence channel (xylenol orange). $\mathrm{h}$ and k: Merged images. I: Differential analysis of the red/green ratio of labelled areas $(\mu \mathrm{m} 2)$ from control and patellectomy sample sections of 5 animals, demonstrating a highly significant difference between new bone and old bone $(* * p<0.01)$. Bar scale: $200 \mu \mathrm{m}$. $429 \times 401 \mathrm{~mm}(300 \times 300 \mathrm{DPI})$ 

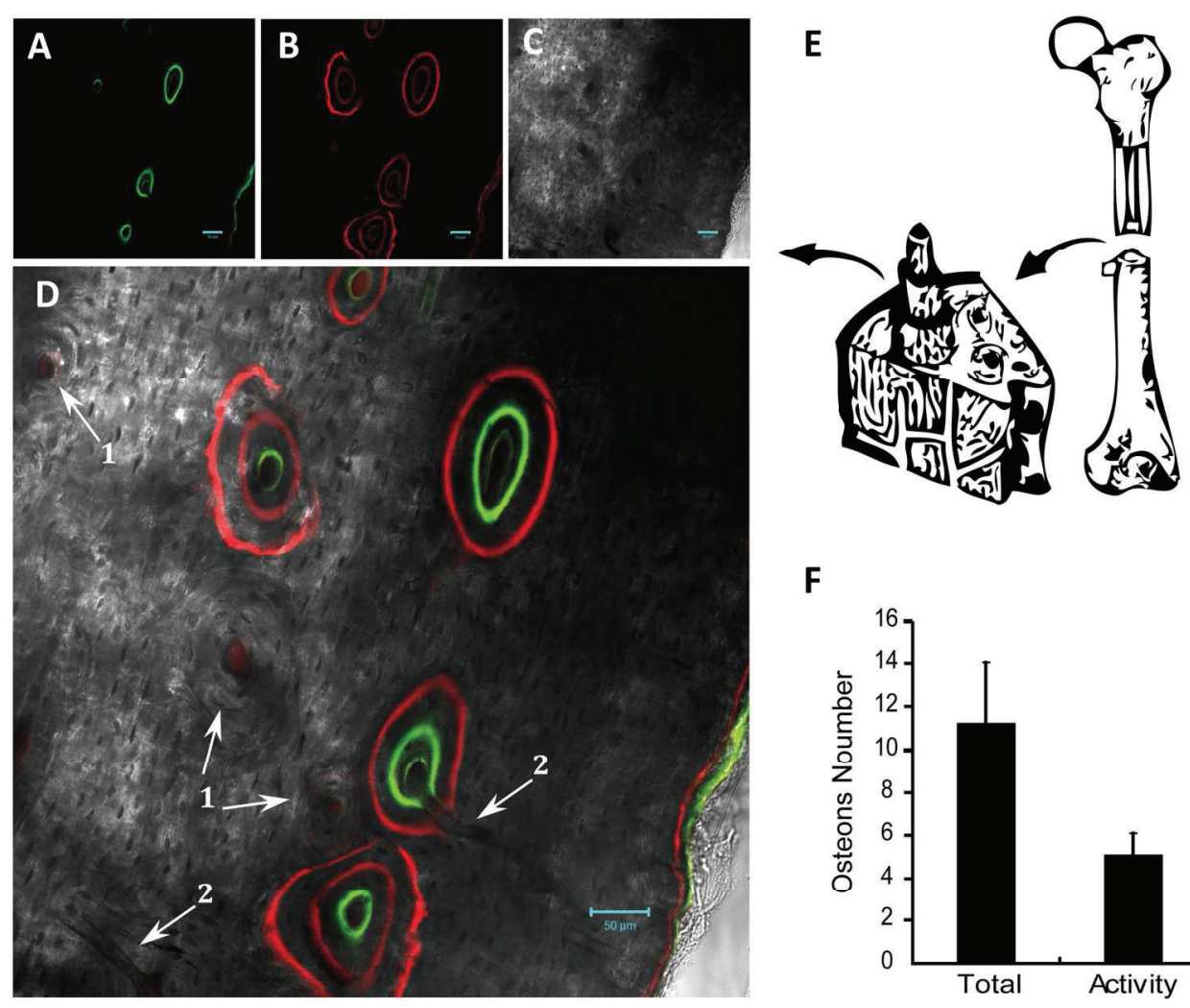

F

Figure 3. Fluorescent images acquired by CLSM plus DIC scanning. Crosscutting section of goat tibia with a thickness of $400 \mu \mathrm{m}$ in thick bone with a scale of $50 \mu \mathrm{m}$. a: Image acquired by green fluorescence channel. b: Image acquired by red fluorescence channel. c: Image acquired by DIC channel. d: Merged image of threechannel CLSM images (green and red fluorescence +DIC) of A, B and C. The merged image shows 2 calcified fluorescence labels from different times (green and red) plus DIC (grey). The weak red color from the second calcein labeling still exist and merged into first green labeling, combined into an yellowish color with various intensities depending upon the ratio of the two colors at developmental stages of those osteons. The arrow 1 indicates quiescent osteons in comparison to the fluorescently labeled active osteons; arrow 2 points out the Volkmann's channels. e: A diagrammatic illustration of the anatomical processing of bone specimens. $\mathrm{f}$ : Mean of total osteon numbers versus mean of the active osteon numbers showed on 10 images (views) from 5 animals at $20 \times$ magnification labeled with fluorescent dyes and acquired by CLSM combined with DIC. $157 \times 129 \mathrm{~mm}(300 \times 300$ DPI $)$ 


\section{University Library}

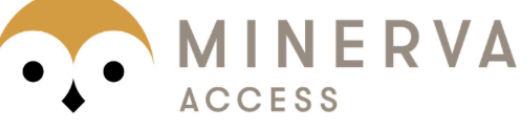

A gateway to Melbourne's research publications

Minerva Access is the Institutional Repository of The University of Melbourne

Author/s:

Yang, X;Qin, L;Liang, W;Wang, W;Tan, J;Liang, P;Xu, J;Li, S;Cui, S

Title:

New Bone Formation and Microstructure Assessed by Combination of Confocal Laser Scanning Microscopy and Differential Interference Contrast Microscopy

Date:

2014-03-01

Citation:

Yang, X., Qin, L., Liang, W., Wang, W., Tan, J., Liang, P., Xu, J., Li, S. \& Cui, S. (2014). New Bone Formation and Microstructure Assessed by Combination of Confocal Laser Scanning Microscopy and Differential Interference Contrast Microscopy. CALCIFIED TISSUE INTERNATIONAL, 94 (3), pp.338-347. https://doi.org/10.1007/s00223-013-9815-6.

Persistent Link:

http://hdl.handle.net/11343/282871 